\title{
Competencias en el ámbito de las Tecnologías de Información y Comunicación que poseen los docentes de Física a nivel universitario
}

\author{
Competences in the field of Information and Communication Technologies \\ held by physics teachers at the university level
}

\section{Resumen}

El objetivo general de esta investigación fue describir las competencias en el ámbito de las Tecnologías de Información y Comunicación (TIC) que poseen los docentes de Física del Instituto Universitario de Tecnología de Valencia (IUTVal). El estudio se sustentó las Competencias Básicas hacia el uso de las TIC establecida por el investigador Pere Marqués. La investigación fue de tipo descriptivo, con un diseño de campo no experimental y transeccional. La población estuvo conformada por seis docentes de la asignatura Física de las cuatro especialidades académicas del IUTVal. Para la recolección de la información, se utilizó el instrumento elaborado por las investigadoras Aguilar y Goncalves (2014). Éste consistió en un cuestionario dicotómico de diecisiete ítems validado bajo un juicio de experto. Del análisis de los resultados se evidencia más del $50 \%$ de los docentes encuestados poseen los conocimientos, habilidades, destrezas y actitudes para desarrollar actividades pedagógicas mediadas con las TIC.

\footnotetext{
Abstract

The general objective of this research was to describe the competences in the field of Information and Communication Technologies (TIC) that the Physics teachers of the Valencia University Institute of Technology (IUTVal) possess. The study was based on the Basic Competences towards the use of TIC established by the researcher Pere Marqués. The research was descriptive, with a non-experimental and transectional field design. The population consisted of six teachers of the Physical subject of the four academic specialties of the IUTVal. To collect the information, the instrument developed by the researchers Aguilar and Goncalves (2014) was used. This consisted of a seventeen item dichotomous questionnaire validated under expert judgment. From the analysis of the results, more than $50 \%$ of the teachers surveyed have the knowledge, abilities, skills and attitudes to develop pedagogical activities mediated with TIC.
}

Palabras clave:

Competencias; física; Tecnologías de Información y Comunicación

\section{Keywords:}

Competences; Physical; Information and Communication Technologies 


\section{INTRODUCCIÓN}

L os avances tecnológicos y los hallazgos acontecidos durante estos últimos años en el mundo de las ciencias, han conllevado a la sociedad del siglo XXI a ser considerada la Sociedad de la Información, del Conocimiento y el Aprendizaje. Así, Zúñiga (2012) expresa que la Sociedad del Conocimiento es consecuencia de los grandes progresos sociales y tecnológicos suscitados en el campo de la información y la comunicación. Es indudable que esta declaración ha direccionado a los sistemas educativos de los países a plantearse reformas y propuestas curriculares que permitan establecer el perfil idóneo del ciudadano digital. Actualmente, la propuesta educativa con mayor aceptación por parte de las naciones del mundo, para asumir este compromiso, lo lidera la formación por competencias. "Toda persona, en cualquier etapa de su vida, debe disponer de oportunidades de aprendizaje permanentes, a fin de adquirir los conocimientos y las competencias necesarias para hacer realidad sus aspiraciones y contribuir a la sociedad" Organización de las Naciones Unidas para la Educación, la Ciencia y la Cultura, UNESCO (Foro Mundial Sobre la Educación, 2015).

Es necesario aclarar que el término competencia posee una amplia variedad de significados. Aunque con diferentes acepciones, todas aluden a conocimientos, habilidades y valores relacionados con la autonomía en la vida del ser humano. Es un concepto complejo muy relacionado con el mundo profesional y que, visto desde una perspectiva reduccionista, es sinónimo de capacidad, idoneidad, habilidad o maestría. Además, éste debe conllevar un saber actuar que permita articular, comparar, dosificar e integrar los recursos adecuados para obtener un resultado satisfactorio (Martínez, 2009).

Entre las competencias propuestas para asumir los desafíos planteados por la sociedad del conocimiento y el aprendizaje, se manifiestan las del ámbito Tecnológico. Algunas investigaciones las refieren como competencias digitales o competencias en el ámbito de las Tecnologías de Información y Comunicación (TIC). No existe en concreción una definición universal. Sin embargo, Zabalza (citado en Hernández, Gamboa y Ayala; 2014) las interpreta como el uso didáctico de las TIC, es decir: un saber qué, un saber hacer y un saber ser, cuando éstas entran en el aula de clase. Por tanto, la 
variedad de recursos didácticos que ofrecen estas tecnologías tienen mucha incidencia en la enseñanza.

Entre tanto, debe considerarse que hoy en día el estudiante de cualquier nivel académico ya no es un simple espectador y consumidor de información. Esta nueva generación (Nativo Digital) se está desprendiendo de la tradicional e inadecuada manera de utilizar la Web 2.0 para asumir el protagonismo de su propia experiencia de aprendizaje, transformándose en un sujeto activo capaz de crear sus propios conocimientos, publicar opiniones, escritos, recursos multimedia, buscar información e inquietudes para luego ser compartida con sus pares a través de las redes sociales. Urge, así pues, la necesidad en que sea el docente el primero en dominarlas e integrarlas a su praxis pedagógica con el fin de orientar a las nuevas generaciones en su adecuado y responsable uso ante la sociedad.

A este hecho, añade Area (2009) que las nuevas generaciones se desarrollan y diferencian entre sí, en la medida de su adelanto educativo y tecnológico. Para que las futuras generaciones tengan éxito en esta nueva sociedad, sociedad de la creatividad, de la innovación y del conocimiento; no sólo deberá bastarles el manejo de las tecnologías, sino que también deberán aprender a usarla con responsabilidad, a pensar de manera creativa, ser reflexivos y críticos constructivos, ser interactivos con el medio, trabajar colaborativamente con su par, éticos y responsables y, sobre todo, ser capaces de comunicar sus planteamientos.

Al respecto, Hernández y Trujillo (2016) manifiestan que uno de los aspectos esenciales al momento de integrar las TIC al ámbito educativo, lo constituye la formación docente. Proceso que debe desarrollarse en las instituciones educativas a través espacios acordes que permitan a los docentes desarrollar competencias tecnológicas en función pedagógica dentro del aula. Para Trujillo y Raso, citados en Hernández y Trujillo (2016), el docente de estos nuevos tiempos debe ser capaz, no solo de diseñar y buscar materiales curriculares con apoyo de las tecnologías; sino también capaz de generar su traslación didáctica y establecer relaciones interdisciplinares entre las diversas áreas del conocimiento que integran el currículo, con el fin de presentar una visión globalizada del mismo; lo que indudablemente lo conllevaría a convertirse en un agente generador de contenidos digitales y diseñador de materiales educativos. 
Por otra parte, cabe destacar que el estudio de la Física ofrece posibilidades potenciales al desarrollo cognitivo de las personas. En primer lugar, es una disciplina que hace referencia a elementos presentes en la naturaleza y a fenómenos cuyos comportamientos hacen que la capacidad de raciocinio y abstracción de las personas desarrollen un ciclo de acción recíproca en campos de conocimientos que exigen, por parte de los que aprenden, la interacción con el objeto mismo de estudio a partir de una práctica diseñada para tal fin. En segundo lugar, permite trabajar los procesos de pensamiento y metacognición con el fin de fortalecer la capacidad creadora y de análisis frente a la producción, uso y asimilación da las tecnologías, cuyos desarrollos están fundamentados, en su mayoría, en los avances científicos de la Física (Castiblanco y Vizcaíno, 2008).

Cabe considerar entonces, que el uso de las TIC pudiera contribuir de manera significativa al proceso de enseñanza y aprendizaje de la Física desde una perspectiva práctica y constructivista. Las aplicaciones prácticas suponen el uso de herramientas tales como los llamados laboratorios virtuales y reales asistidos por computadoras, los proyectores de videos, los programas ofimáticos, la Realidad Aumentada (RA), los televisores, los teléfonos móviles de alta gama, las Tablets, entre otros; todo con el fin de mostrar a los estudiantes el desarrollo de un fenómeno o proceso que por sí solo no tendría significado alguno. En cuanto a la aplicación constructivista, media la investigación, la innovación, la socialización y la creación de portales web, técnicas que, estratégicamente bien empleadas, dan sentido significativo a la enseñanza de la Física. Acevedo y otros (citados en Cuellar, Salazar, Alvear, Marín, Calderón, Díaz, Naveros, Cardoso, Castro, Pascuas, Bautista y Gutiérrez; 2017).

En tal sentido, y considerándose todo lo expuesto hasta ahora, las potencialidades que ofrece el estudio de la física al conocimiento humano puede potenciarse si se les combina adecuadamente con las TIC. Sin embargo, existen obstáculos que impiden este cometido tales como el desconocimiento, inadecuada formación, desinterés, inexperiencia o el temor que algunos docentes manifiestan hacia el uso de las mismas. Esto representa un problema ya que los sistemas educativos a nivel mundial están experimentando transformaciones innovadoras a través del uso de las TIC y su desconocimiento por parte del docente sería un aporte más a la brecha digital. Es decir, la separación que existe entre las personas que utilizan las TIC como 
parte rutinaria de su vida diaria, de aquellas que no tienen acceso a las mismas o que simplemente las tienen, pero no saben usarlas (Ortiz, 2012).

De modo que, la innovación tecnológica en educación no significa únicamente la incorporación del recurso tecnológico en el aula, implica una transformación cultural en la manera de gestionar y construir el conocimiento de forma didáctica e interactiva. Lugo y Kelly (citados en Competencias TIC para el Desarrollo Profesional Docente, 2013). El uso innovador que se le dé a las TIC y su adecuada instrumentación tanto a lo interno como a lo externo del aula para crear experiencias significativas de aprendizaje; dependerá en gran parte del docente y de las políticas de formación y actualización que lleve a cabo la institución educativa.

Venezuela actualmente cuenta con un marco legal y con instituciones ministeriales abocadas a planes y proyectos TIC con el objeto de incorporarlas a la sociedad. En el caso de Venezuela la inclusión de las TIC, en el ámbito educativo, se encuentran orientadas a la formación integral del ciudadano mediante el aprendizaje liberador mediado por el uso de las Tecnologías Libres (TL). El Proyecto Canaima Educativa y recientemente el Proyecto Canaima Universitaria, representan dos de estas iniciativas impulsadas por el Estado en pro de coadyuvar a ese proceso. Sin embargo, la falta de formación de algunos docentes en la utilización didáctica de las Laptop, Tablets y programas, han originado que la pedagogía tradicional siga arraigada en las aulas. Dejando a un lado la verdadera innovación educativa y dando paso al mero uso instrumental de los equipos. En opinión de Cabero (2005) el mal uso que se le da a las TIC, se debe en gran parte a políticas educativas poco claras con respecto a planes y programas de formación.

Desde el ámbito de la educación universitaria, en el país se están ofertando programas y carreras académicas a distancia, tal es el caso de la Universidad Central de Venezuela que bajo el esquema de Estudios Universitarios Supervisados (EUS) ofrece las carreras de Educación e Ingeniería de Procesos Industriales a distancia, en la modalidad virtual a partir del segundo semestre, núcleo Cagua (Aragua), Campus Virtual UCV (2016). La Universidad Lisandro Alvarado de Barquisimeto (UCLA) con el curso a distancia Métodos de Investigación y Vigilancia Epidemiológica en Salud Ocupacional a cargo del profesor Parra, H. Enfoque Ocupacional (2016) y la Universidad de Carabobo (UC) con el entorno virtual SEDUC 
(Sistema de Educación a Distancia de la UC) con diplomados y programas orientados a la formación docente en TIC. No obstante, debe advertirse de manera reiterativa que la calidad de la teleeducación o cualquiera otra innovación, no reside en el uso de las tecnologías de la información; sino en la formación, intención y decisión del docente más que el de un experto informático (García, Ruiz y Domínguez, 2007).

Hasta acá, queda más que señalada la necesidad y el compromiso de desarrollar en los docentes activos y en formación una serie de competencias básica enmarcadas en la capacidad de diseñar experiencias de aprendizaje significativas en los estudiantes a través del uso oportuno y eficaz de las TIC. La relación existente entre el acto pedagógico y las competencias TIC; representan la posibilidad de trascender la tradicional práctica pedagógica bancaria y transmisora de conocimiento, a una en donde el estudiante sea el participe y generador del mismo.

Para los estudiantes del IUTVal, los contenidos de Física revisten fundamental importancia a la consolidación de sus competencias específicas. Teniendo en cuenta que hoy en día las TIC están presentes en todas las áreas del conocimiento, resulta paradójico que una institución en donde se enseña ciencia y tecnología, parte del personal docente de Física no haga uso de ellas. Observaciones realizadas por el investigador en esta institución universitaria, ubicada en el Estado Carabobo, municipio valencia, parroquia San José; arrojaron ciertos indicios de inexistencia de políticas educativas y programas de formación TIC hacia el profesorado de esta casa de estudio. El investigador presume que posiblemente los docentes no estén capacitados; que exista desconocimiento, por parte de éstos, en cuanto al uso de las TIC en la enseñanza de la Física o que carezcan de las herramientas. Es aquí donde se plantea la necesidad de conocer las causas de este problema y preguntar: ¿Cuáles son las competencias que en el ámbito de las Tecnologías de Información y Comunicación (TIC) poseen los docentes de Física del Instituto Universitario de Tecnología de Valencia (IUTVal)?

De esta pregunta surge el objetivo general estructurado de la siguiente manera: Describir las Competencias en el ámbito de las TIC que poseen los docentes de Física del IUTVal. Luego para poder llegar a dicho objetivo se reestructuraron los siguientes objetivos específicos: 1. Diagnosticar las competencias técnicas instrumentales TIC que poseen los docentes de Física. 2. Determinar 
la actualización profesional de los docentes de Física con respecto a las TIC. 3. Identificar la metodología de trabajo que usan los docentes de Física con las TIC. 4. Precisar la actitud de los docentes de Física hacia el uso de las TIC, en el contexto educativo.

Algunas investigaciones referentes al tema, son las siguientes:

Los profesores Hernández y Trujillo (2016), ambos adscritos a la Facultad de Educación de la Universidad de Granada, España, realizaron, como parte de un trabajo doctoral, una investigación referente al proceso de enseñanza mediado con tecnología educativa denominada Competencias Docentes en el Uso y Aplicación de las TIC en San Juan Girón (Colombia). Esta investigación consistió en pretender el porcentaje de docentes con competencias TIC y cuántos de ellos las aplicaban en las aulas. Fue una investigación de campo, cuantitativa y descriptiva, en la que se aplicaron cuestionarios y entrevistas a rectores, coordinadores, profesores y estudiantes que hacen parte del sistema educativo público del municipio. Sobre los datos, se realizaron pruebas no paramétricas de correlaciones, tablas de frecuencia e histogramas, así como el uso de la estadística descriptiva bidimensional para cuantificar el grado de asociación que existía entre las variables en estudio. Los resultados obtenidos relacionaron los porcentajes de las competencias TIC de la población en cuestión; arrojando como resultado un bajo porcentaje de docentes con competencias TIC en el sector educativo del municipio Girón y poca aplicabilidad en las aulas.

Los investigadores igualmente determinaron que la comunidad educativa del municipio Girón tenía conocimiento de la existencia de las herramientas TIC pero que, debido a la carencia de planes efectivos de capacitación en su uso, las mismas no habían podido ser empleadas pedagógicamente en las aulas.

Por su parte, la revista on line multidisciplinaria Dialógica de la Universidad Pedagógica Experimental Simón Bolívar (UPEL) Venezuela. Publicó en el primer semestre del año 2017, la investigación titulada Las Tic en el Desempeño del Docente en el aula, una mirada al Liceo Bolivariano Presbítero "Luis María Sucre". Investigación conducida por Pineda y Wilinski. Fue un estudio de tipo cualitativo y enmarcado el método hermenéutico para interpretar la información recabada. Esta compilación de datos e información se realizó a través de una entrevista semiestructurada aplicada a tres docentes de la institución educativa ya mencionada. El propósito fundamental fue conocer la visión que tienen los docentes con 
respecto al uso pedagógico de las TIC dentro del aula. La información recabada fue categorizada y triangulada. En conclusión, el estudio evidenció que los docentes se mostraron entusiastas hacia el uso de las TIC, pero por falta de conocimiento y de lineamientos claros para su apropiación y utilización, no las empleaban, a expensa de contar con la infraestructura y los equipos.

Cabero y Marín (2014) presentaron, en la revista on line venezolana Enl@ce, un artículo en el cual hacen un análisis sobre los programas de formación en TIC que, a juicio de los expertos citados, deben reunir tales iniciativas. El artículo denominado Miradas sobre la Formación del Profesorado en Tecnologías de Información y Comunicación (TIC) pretende un análisis y revisión crítica de las fundamentaciones teóricas y conceptuales del tema en cuestión. Un análisis sustentado en una investigación de carácter documental y descriptivo que permite considerar los fundamentos necesarios para dar una proyección innovadora con respeto al tema. Para los expertos, las dimensiones y características, desde la cual se abordan las TIC, no deben ser dirigidas como actividades puntuales, ocasionales e instrumentales. Que el docente no adquiera sólo el manejo y compresión de las TIC, sino que comprenda que las TIC van a permitir desarrollar y ejecutar actividades diferentes. Por tanto, deben cambiarse las formas y métodos de emprender el uso de las TIC para lograr entornos diversos e interactivos que permitan su inserción en las aulas.

Por su parte Mora (2015) elaboró un trabajo de investigación que tuvo como propósito comprender la actitud de los docentes ante el uso de las TIC en el Marco del Proyecto Canaima Educativa en la U. E. Colegio Don Bosco del municipio Valencia del Estado Carabobo. Estudio enmarcado en el paradigma Postpositivista, de tipo cualitativo, método etnográfico y diseño descriptivo. La población en estudio estuvo conformada por seis (6) docentes de las diferentes áreas de conocimiento de la institución. Se empleó el Diario de Campo como instrumento de recolección de información obtenida a través de la observación participante, el guion y la entrevista semiestructurada. La investigación concluyó en demostrar que la falta de instrucción y desconocimiento, que tienen los docentes, sobre el uso de las TIC (más allá de las posturas ideológicas o personales de cada docente) les genera rechazo, y en muchas ocasiones angustia cuando se les exige su implementación en las aulas de clase. Se manifiesta claramente que el Proyecto Canaima 
Educativa adolece de políticas clara que permitan girar lineamientos e instrucciones que orienten su correcta implementación en el sistema educativo.

Todos los autores, acá citados, convergen en señalar sobre la necesidad de capacitar, en primera instancia, a los docentes hacia el uso de las TIC antes que éstas sean incorporadas a los salones de clase. Los programas de formación, que a bien deban ser incorporados en las instituciones educativas, indistintamente del nivel académico que representen, deben reunir ciertas características adecuadas a las necesidades de cada realidad educativa. Para que los docentes logren hacer de su praxis educativa un proceso de enseñanza aprendizaje de óptima calidad, exigencia de este siglo, deberán avocarse a sus competencias tecnológicas, sino desea ser parte del obsoletísimo histórico.

En tal sentido, la sociedad de este siglo reclama constantemente la capacidad de localizar, comprender, analizar, aplicar y relacionar los diferentes datos a los que tiene acceso para convertirlos en conocimiento. En palabras de Majó (2000) "será una habilidad imprescindible saber seleccionar, filtrar, ordenar, valorar y asimilar la información para poderla convertir en conocimiento útil” (p. 77). No se trata de saber más, sino de tener las competencias necesarias para acceder y transformar esos contenidos en SABER. Por ello, la educación resulta el medio idóneo para alcanzar tal objetivo. Un derecho que va más allá de la simple formación inicial, a una que la garantice a lo largo de toda la vida.

La UNESCO en aras de contribuir a la calidad de los sistemas educativos, económicos y sociales de los países miembros. Propuso en el 2008 un marco de referencia de Estándares de Competencias en TIC para Docentes (EDC-TIC) desde el cual se ofrecen orientaciones a todo los docentes y más concretamente directrices para planear programas de formación docente y cursos que permitirán prepararlos para desempeñar un papel esencial en la capacitación tecnológica de los estudiantes. En fin, el proyecto EDCTIC busca mejorar la praxis de los docentes combinando para ello las competencias TIC con lo nuevo de la pedagogía, más el propósito que tengan los docentes de incorporarlas en las aulas.

De lo anteriormente planteado se deduce que la Educación es un Derecho humano que debe mantenerse a lo largo de la existencia humana. Debe ser una educación inclusiva y de calidad. Siendo la UNESCO la única organización de las Naciones Unidas (ONU) 
autorizada a nivel mundial para abarcar todo lo concerniente a la Educación; se le confió en el 2016 la coordinación de la agenda: Educación Mundial 2030 en el ámbito del Objetivo Sostenible de Desarrollo (ODS) 4 el cual consiste en: "Garantizar una educación inclusiva, equitativa y de calidad y promover oportunidades de aprendizaje durante toda la vida para todos".

\section{MÉTODO}

$\mathrm{T}$ odo estudio científico requiere de un orden metodológico que permita dar coherencia, fiabilidad, objetividad y validez a los hechos que analiza. Por este motivo, el marco metodológico representa la parte práctica y neurálgica de la investigación. Ésta se encuentra representada por una serie de pasos que el investigador debe definir y ejecutar para dar respuesta a las diferentes interrogantes objeto de investigación. Estos pasos incluyen la definición del tipo y diseño de la investigación abordada, los sujetos que estarán sometidos a estudio y las técnicas e instrumentos que se emplearán para la recolección de los datos, incluyendo la determinación de validez y confiabilidad del instrumento a ser empleado. (Escobar, Liévano, Marchán, Ramírez I, Ramírez J, 2013).

El presente estudio se enmarcó dentro del diseño investigativo no experimental, tipo campo, nivel descriptivo transeccional. Es decir, no experimental tipo campo significa que el investigador no manipula de manera deliberada las variables. "Observa los hechos tal y como se presentan en su contexto real" (Palella y Martins, 2010, p. 87). Descriptiva, porque caracteriza el fenómeno con el fin de establecer su estructura o comportamiento (Arias, 2012), y transeccional porque la información se recolecta en un solo momento, en un tiempo único (Hernández, Fernández y Baptista, 2010) tal y como se tomaron dentro del contexto universitario del Instituto Universitario de Tecnología de Valencia.

Las técnicas de recolección de datos representan el vínculo directo con el objeto de investigación y el campo de trabajo. Las técnicas de recolección de datos, representan las distintas formas o maneras de obtener información del terreno de estudio (Palella y Martins, 2010). Para la presente investigación se escogió como técnica de recolección de información la Encuesta. "La Encuesta es una técnica destinada a obtener datos de varias personas cuyas 
opiniones interesan al investigador." (Palella y Martins, 2010, p.123)

El Instrumento de recolección de información representa el con qué se va a recoger, filtrar y codificar la información. Palella y Martin (2010) la definen como "recurso del cual puede valerse el investigador para acercarse a los fenómenos y extraer de ellos información". Como instrumento de recolección de información se utilizó el cuestionario; el cual consiste en una comunicación escrita básica entre el encuestador y el encuestado en base a un conjunto de preguntas relacionadas con el evento de estudio. (Hurtado de Barrera, 2010).

El cuestionario utilizado en esta investigación corresponde al empleado por Aguilar y Goncalves, quienes en el año 2014 presentaron un Trabajo Especial de Grado similar al propuesto por el autor, con la notoriedad en que el usado por las investigadoras mencionadas estuvo dirigido a docentes de la Asignatura Cálculo III de la Facultad de Ciencias de la Educación de la Universidad de Carabobo. En esta oportunidad el instrumento estuvo dirigido a seis (6) docentes de la Asignatura Física pertenecientes al gremio de profesores del Instituto Universitario de Tecnología de Valencia. El cuestionario se aplicó de forma personal y constó de diecisiete (17) ítems o preguntas cerradas dicotómicas, entendiéndose éstas como aquellas que ofrecen dos (2) opciones de respuestas: Sí o No (Arias, 2012). Añadido a las respuestas se halla la justificación, con el fin obtener datos que permitan realizar análisis de los resultados.

El instrumento empleado en esta investigación se validó en el 2014 a través del juicio de cinco (5) expertos, integrado por docentes pertenecientes al Departamento de Matemática y Física, y al Departamento de Computación de la Facultad de Ciencias de la Educación de la Universidad de Carabobo.

En lo que respecta a la confiabilidad, grado en que un instrumento produce resultados consistentes y coherentes cuando se aplica repetidamente al mismo sujeto en diferentes circunstancias (Claret, 2005). Éste no pudo ser calculado debido a lo pequeño de la población. En total, seis (6) docentes de Física representan la población objeto de estudio.

Esta sección, siguiendo a Arias (2012), especifica las diferentes etapas a la que fue sometida la información recabada: registro, clasificación, tabulación, codificación e interpretación. 
Los datos fueron agrupados y ordenados en una matriz para luego ser graficados mediante el programa ofimático Excel 2016 para Windows de Microsoft. Una vez agrupados y ordenados, se aplicaron los estadísticos correspondientes para proceder a describir cuantitativamente el fenómeno. El análisis cualitativo se llevó a cabo mediante la interpretación de los resultados obtenidos del análisis cuantitativo de los encuestados, elaborando para ello un texto narrativo por cada dimensión abordada en la investigación.

\section{RESULTADOS Y DISCUSIÓN}

$\mathrm{D}$ entro del análisis y presentación de los resultados derivados de la aplicación del instrumento de recolección de información: El cuestionario. De acuerdo con Palella y Martins (2010), la interpretación de los resultados permite resumir y sintetizar la información recabada, a objeto de proporcionar mayor claridad a las respuestas y conclusiones respecto a las inquietudes, dudas o interrogantes planteadas en la investigación. A propósito de lo antes expuesto, Selltiz, Jahoda y otros (citados en Balestrini, 2002) reafirman que "El propósito del análisis es resumir las observaciones llevadas a cabo de forma tal que proporcionen respuestas a las interrogantes de investigación" (p. 169).

Es una etapa de carácter técnico, de mucha reflexión que involucra la incorporación de ciertas operaciones ordenadas y coordinadas que facilitarán la interpretación significativa de los datos, todo en correspondencia con las bases teóricas que orientan la investigación. Comprende además la codificación, la tabulación, las técnicas de presentación y el análisis estadístico que a bien corresponda al estudio emprendido. Reafirmando lo antes expuesto, Balestrini (2002) manifiesta que "el análisis e interpretación de los datos, se convierte en la fase de la aplicación de la lógica deductiva e inductiva en el desarrollo de la investigación." (p.170)

Para el análisis e interpretación de los resultados, se diseñaron dos tipos de matrices de tabulación y codificación por ítems, la cual contienen para cada docente el tipo de respuesta seleccionada. Una está referida al análisis por dimensión y la otra referida a la tendencia de la justificación aportada por el encuestado en cada ítem. El análisis estadístico se basó en una escala de medición nominal, siendo los estadísticos utilizados: Distribución de frecuencia $(\mathrm{F})$, proporciones $(\mathrm{Pi})$, porcentajes (\%) y media aritmética. 
Los resultados fueron recabados a través de la aplicación de un cuestionario de carácter dicotómico (Ver Anexo B) a seis (6) docentes de la asignatura Física del Instituto Universitario de Tecnología de Valencia (IUTVal). De igual manera, se les solicitó a los docentes justificasen sus respuestas seleccionadas. De esta forma las respuestas obtenidas fueron:

a) S (Sí, sin justificación)

b) N (No, sin justificación)

c) SJ (Sí, con justificación)

d) NJ (No, con justificación)

e) J (Sólo justifica)

Las totalizaciones de los resultados obtenidos de la encuesta aplicada, se muestran resumidos en la matriz de Presentación de Resultados (Ver Cuadro 2, p. x). En ésta puede observarse las frecuencias totales obtenidas del tipo de respuestas arrojadas por el cuestionario: S, N, SJ, NJ y J. En la representación gráfica, se dispuso para el análisis de las dimensiones el diagrama circular sectorizado (diagrama de torta) en virtud del número de categorías que representa la respuesta: Sí y No. Para el análisis de la tendencia a la justificación se dispuso del diagrama de barra.

\section{CONCLUSIONES}

$\mathrm{D}$ e acuerdo con los resultados obtenidos en este estudio, se concluye que más del $50 \%$ de los docentes de Física del Instituto Universitario de Tecnología de Valencia, poseen los conocimientos, habilidades, destrezas y actitudes para desarrollar actividades pedagógicas a través de las Tecnologías de Información y Comunicación como estrategia de enseñanza y de aprendizaje.

Es en la Dimensión Metodología Docente donde, a pesar de poseer los docentes conocimiento sobre el tema, se detalla la deficiencia. Al ser analizadas las justificaciones de los ítems correspondientes a esta dimensión, los mismos apuntaron a la falta de herramientas, recursos, ausencia de logística, conectividad e infraestructura. 
La mayor parte de los docentes encuestados manifiestan su buena disposición al uso de las TIC como estrategia de aprendizaje y enseñanza de la Física. Expresan tener una Actitud positiva al reconocer la importancia que para la educación reviste la actualización profesional en materia de innovación tecnológica aplicada al ámbito pedagógico.

En lo que respecta a la actualización profesional, la mayor parte de los docentes encuestados poseen estudios relacionados con las TIC y con innovaciones tecnológicas en sus áreas de especialización. En general, son ingenieros y licenciados en ciencia duras dedicadas a la docencia que, de alguna u otra manera, han sabido combinar su especialidad con la pedagogía. En tal sentido, no es de extrañarse el resultado obtenido en este estudio al pretender describir las competencias que en el ámbito de las TIC poseen.

Establecer mesas de trabajo en la cual se discutan planes y propuestas en pro de mejorar e incorporar las TIC a la Metodología Docente, no sólo para la enseñanza de la Física, sino también para otras asignaturas contenidas en la pensa de los egresados. Para ello deberán solicitar a las autoridades respectivas la reapertura del Departamento de Tecnología Educativa.

Se requiere emprender desde la academia un estudio serio y objetivo a miras de mejorar y adecuar los espacios de la institución con el objetivo de integrar las TIC al aula. El docente debe disponer de equipos y personal capacitado que le oriente e instruya sobre el tema.

Existe la disposición y el personal. Así quedó demostrado en este estudio que aun cuando estuvo dirigido sólo a seis docentes de la asignatura de Física, es un punto de inicio para adecuar y actualizar al personal docente del Instituto Universitario de tecnología de Valencia (IUTVal) a la innovación pedagógica con el uso de las tecnologías.

\section{REFERENCIAS}

Aguilar, S. y Goncalves, A. (2014). Descripción de las Competencias Tecnológicas en Información y Comunicación (TIC) que poseen los Docentes de la Asignatura Cálculo III, Mención Matemática de la Facultad de Ciencias de la Educación de la Universidad de Carabobo. (Trabajo Especial de 
Grado no publicado). Recuperado de http://www.riuc.bc.uc. edu.ve/bitstream/123456789/1559/3/4711.pdf

Area, M. (2009). Introducción a la Tecnología Educativa (Manual). Recuperado de http://es.slideshare.net/Nurieh/introduccion-ala-tecnologia-educativa

Arias, F. (2012). El Proyecto de Investigación. Introducción a la Metodología Científica. (5o ed.). Caracas: Episteme C. A

Balestrini, M. (2002). Como se elabora el Proyecto de Investigación. ( $6^{\mathrm{a}}$ ed.). Caracas: BL Consultores y Asociados Servicio Editorial

Cabero, A. (2005). Estrategias para la Formación del Profesorado en TIC. Universidad de Sevilla, España. Recuperado de http://mc142.uib.es:8080/rid=1JGJCFRRT-1X608XLLM1/CABERO\%20TEMA1.pdf

Cabero, J. y Marín, V. (2014). Miradas sobre la formación del profesorado en Tecnologías de Información y Comunicación (TIC). Revista Venezolana de Información, Tecnología y Conocimiento (Enl@ce): 11 (2), 11-24. Recuperado de http://www.redalyc.org/html/823/82332625005/

Castiblanco, L. y Fabián V. (2008). Uso de las TICs en la Enseñanza de la Física. pp. 20-26 Recuperado de http://www.unilibre.edu.co/revistaingeniolibre/revista7/articulo s/El-uso-de-las-TICs.pdf

Cuellar, Z., Salazar, S., Alvear, G., Morín, G., Calderón, A., Díaz, M., Naveros, G., Cardoso, H., Castro, M., Pascuas, Y., Bautista, C. y Gutiérrez, N. (2017). Actitud, conocimiento y uso de las Tecnologías de la Información y la Comunicación (TIC) para la Enseñanza de las Ciencias Naturales en las Instituciones Educativas Públicas del Municipio de Neiva: Un estudio diagnóstico. [Revista en línea] Memorias del IX Encuentro Nacional de Experiencias en Enseñanza de la Biología y la Educación Ambiental. IV Congreso Nacional de Investigación en Enseñanza de la Biología. Pp. 1211-1220 Recuperado de http://revistas.pedagogica.edu.co/index.php/bio-grafia/article /view/7292

Escobar, Liévano, Merchán, Ramírez, I. y Ramírez, J. (2013). Manual de Trabajo de Grado para la Carrera de Educación en toda sus Menciones del Programa Académico Santa Bárbara. Recuperado de http://files.unellezsantabarbara.webnode.com .ve/200000118-da9ffdc92c/MANUAL\%20DE\%20T

RABAJO\%20DE\%20GRADO.pdf

García, Ruiz y Domínguez. (2007). De la Educación a Distancia a la Educación Virtual. Barcelona, España: Ariel, S. A

Hernández, C., Glenn y Trujillo T. (2016). Competencias Docentes en el uso y aplicación de las TIC en San Juan Girón (Colombia). Doi: 10.19044/esj.2016.v12n13p51 pp. 51-66 (Trabajo Doctoral) 
Recuperado de http://eujournal.org/index.php/esj/article/ download/7459/7184

Hernández, C. Gamboa, A. y Ayala, E. (2014). Competencias TIC para los Docentes de Educación Superior. Congreso Iberoamericano de Ciencia, Tecnología, Innovación y Educación Buenos Aires, Argentina 12/13 y 14 de noviembre ISBN: 978-84-7666-210-6 - Artículo 837 Recuperado de http://www.scielo.org.co/pdf/prasa/v7n14/v7n14a03.pdf

Hernández, S. Fernández, C. y Bastidas, L. (2010). Metodología de la Investigación. (5o ed.) México, D.F: McGraw-Hill Interamericana

Hurtado de Barrera, J. (2010). El Proyecto de Investigación: Comprensión Holística de la Metodología y la Investigación. (6 ${ }^{a}$ ed.). Venezuela: Fundación Sypal, Ediciones Quirón S.A

Marqués, P. (2014). Competencia Digital Docente. Chispas TIC y Educación. [Mensaje en Blog] Recuperado de http://peremarques.blogspot.com/search/label/COMPETENCIA \%20DOCENTE

Martínez López, F. (2009). Las Tecnologías de la Información y la Comunicación (TIC) y las Competencias Básicas en Educación. Espiral: Cuaderno del Profesorado, 2(3), 15-16. Recuperado de http://www.cepcuevasolula.es/espiral. / https://dialnet.unirioja. es/descarga/articulo/2898369.pdf

Mora, A. (2015). Actitud del Docente ante el uso de las Tecnologías de Información y Comunicación en el Proyecto Canaima Educativa. [Documento en línea] Trabajo Especial de Grado no publicado. Recuperado de http://www.riuc.bc. uc.edu.ve

Organización de las Naciones Unidas para la Educación la Ciencia y la Cultura. (UNESCO) [Foro Mundial Sobre la Educación 2015] 19-22 de mayo de 2015, Incheon, República de Corea. Recuperado de http://webarchive.unesco.org/20160930040522 /http://es.unesco.org/world-education-forum-2015/aboutforum/declaracion-de-incheon

Organización de las Naciones Unidas para la Educación, la Ciencia y la Cultura. UNESCO. (2008). Estándares de Competencias en TIC para Docentes. Recuperado de http://www.oei.es/ tic/UNESCOEstandaresDocentes.pdf

Organización de las Naciones Unidas para la Educación, la Ciencia y la Cultura. UNESCO (2017). Foro Internacional sobre las TIC y Educación 2030. Recuperado de https://en.unesco.org/themes /ict-education/international-forum-2017

Ortiz, R. (2012). La Brecha Digital. Compartiendo conocimiento para un futuro mejor. (OpenMind) Recuperado de https://www.bbvaopenmind.com/la-brecha-digital/ 
Palella, S. y Martins, F. (2010). Metodología de la Investigación Cuantitativa. Caracas: Fondo Editorial de la Universidad Pedagógica Experimental Libertador FEDUPEL

Pineda, S. y Wilinski, A. (2017). Las TIC en el Desempeño Del docente en el Aula Una mirada al Liceo Bolivariano Presbítero " Luis María Brito". Revista Multidisciplinaria Dialógica: 14 (1), 159-170. Recuperado de http://revistas.upel.edu.ve/index.php/ dialogica/issue/view/1/showToc

Zúñiga, R. (2012). Sociedad del Conocimiento y Educación. Noria de Ángeles, Zacateca, México D.F. Recuperado de http://es. slideshare.net/mirella2012/ensayo-sociedad-del-conocimiento-yeducacin-reto-o-derecho 